\title{
State boundary surface in hypoplasticity
}

\author{
David Mašín ${ }^{1}$ and Ivo Herle ${ }^{2}$ \\ 1 Department of Enginnering Geology, Charles University, Albertov 6, 12843 \\ Prague 2, Czech Republic masin@natur.cuni.cz \\ 2 Institute of Geotechnical Engineering, Technische Universität Dresden, 01062 \\ Dresden, Germany ivo.herle@mailbox.tu-dresden.de
}

\section{Introduction}

Hypoplastic constitutive models have been developed since 1980's and since then they have established a solid base for an alternative description of the soil behaviour, without an explicit definition of yield and potential surfaces, see e.g. the review by [15]. Recent hypoplastic models $[3,16]$ include the concept of critical states and have been successfully used in many computations of boundary value problems within coarse-grained soils. The progress of hypoplastic models suitable for the description of fine-grained soils has been delayed. Rate-dependent $[13,4]$ and rate-independent $[6,8]$ hypoplastic models for clays promise to follow the success of the development for sand. Nevertheless, a thorough testing of various constitutive aspects is required in order to ensure a correct performance in general conditions of BVPs.

One of the key characteristics of soil behaviour, incorporated in different ways in the most of the currently available elasto-plastic constitutive models, is the presence of the surface in the stress-porosity space which bounds all accesible states (state boundary surface). Hypolastic models do not incorporate the state boundary surface explicitly in the mathematical formulation. However, as demonstrated by [11] for a particular hypoplastic model for clay [8], state boundary surface is predicted implicitly by the constitutive equation as a by-product of the mathematical formulation.

As shown in [11], state boundary surface of a hypoplastic model for clays is sufficiently accurately approximated by the so-called swept-out-memory surface, a surface in the stress-porosity space which coveres asymptotic states (limit states, attractors) achieved after sufficiently long proportional deformation paths. The purpose of this paper is to provide a mathematical derivation for the limit states of the two particular endomorphous hypoplastic models a hypoplastic model for clays by Mašín [8], which follows from [6] and [13], and a hypoplastic model for granular materials by von Wolffersdorff [16]. 


\section{Basic properties of considered constitutive models}

A complete mathematical formulation of both models considered in the paper is given in Appendices. In its most general form, mathematical formulation of the models is given by

$$
\stackrel{\circ}{\mathbf{T}}=\mathrm{h}(\mathbf{T}, \mathbf{D}, e)
$$

where $\mathbf{T}^{\mathbf{T}}$ is a co-rotated (Jaumann) stress rate, $\mathbf{T}$ stands for the Cauchy's stress tensor, $\mathbf{D}$ is the Euler's stretching tensor and $e$ is void ratio. All stresses are considered as effective in the sense of Terzaghi. According to the terminology laid out by Kolymbas [7], models belong to the sub-class of hypoplastic models referred to as endomorphous. In addition to the Cauchy stress $\mathbf{T}$, void ratio $e$ is also considered as a state variable. The particular form of the isotropic tensor-valued function $\mathrm{h}$, adopted in constitutive model by [8], follows from [3] and reads

$$
\stackrel{\circ}{\mathbf{T}}(\mathbf{T}, \mathbf{D}, e)=f_{s}(\operatorname{tr} \mathbf{T})\left[\mathcal{L}(\hat{\mathbf{T}}): \mathbf{D}+f_{d}(\operatorname{tr} \mathbf{T}, \mathrm{e}) \mathbf{N}(\hat{\mathbf{T}})\|\mathbf{D}\|\right]
$$

where the operator trace is defined by $\operatorname{tr} \mathbf{X}=\mathbf{X}: 1$ with 1 being the secondorder unit tensor, $\hat{\mathbf{T}}$ is the normalised stress defined by $\hat{\mathbf{T}}=\mathbf{T} / \operatorname{tr} \mathbf{T},\|\mathbf{D}\|=$ $\sqrt{\mathbf{D}: \mathbf{D}}$ is the Euclidian norm of $\mathbf{D}$ and the operator arrow is defined as $\overrightarrow{\mathbf{D}}=\mathbf{D} /\|\mathbf{D}\| . f_{s}$ and $f_{d}$ are barotropy and pyknotropy factors $[3,16]$.

The barotropy factor $f_{s}$ of the hypoplastic model for clays [8] is independent of void ratio $e$ and is a linear function of $\operatorname{tr} \mathbf{T}$. It follows that for a constant value of the pyknotropy factor $f_{d}$ (i.e., for swept-out-memory conditions) the model [8] is positively homogeneous of degree 1 with respect to stress, i.e.

$$
\gamma^{2} \stackrel{\circ}{\mathbf{T}}(\mathbf{T}, \mathbf{D}, e)=\stackrel{\circ}{\mathbf{T}}\left(\gamma^{2} \mathbf{T}, \mathbf{D}, e\right)
$$

The barotropy factor of the hypoplastic model for granular materials [16] is a non-linear function of $\operatorname{tr} \mathbf{T}$ and void ratio. For this reason the model is not positively homogeneous with respect to stress. However, as discussed in [14], for a constant value of $f_{d}$ the model is directionally homogeneous

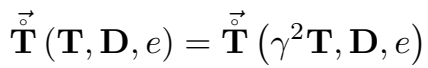

which is a sufficient condition to predict asymptotic behaviour [14].

\section{Proportional stress and strain paths}

Behaviour along proportional stress and strain paths (with $\overrightarrow{\mathbf{D}}$ and $\overrightarrow{\mathbf{T}}$ being constant) is reflected in the so-called swept-out-memory (SOM) states, see Fig. 1. These SOM-states can also be considered as attractors of the soil behaviour [2]. The response of a real soil, however, does not depend solely 

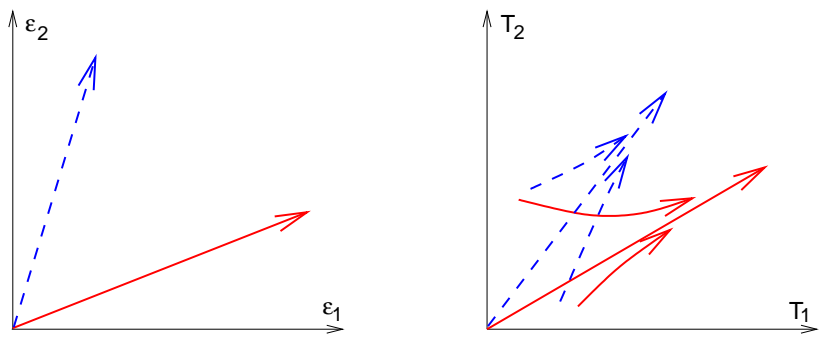

Fig. 1. SOM-behaviour: proportional stress paths for proportional strain paths

on the stress tensor. Void ratio must be considered as an additional state parameter for both coarse-grained and fine-grained soils.

Except for constant volume (undrained) deformation, void ratio changes along proportional strain paths. In case of hypoplastic models for pairs of proportional stress and strain paths one can find corresponding void ratios $e_{p}$ dependent on the mean stress $p$. Combinations of $e_{p}$ and $p$ for $\operatorname{tr} \overrightarrow{\mathbf{D}}<0$ plotted in the $e: p$ space can be denoted as normal compression lines. Their positions in the $e: p$ space may be characterised by the void ratio at vanishing mean stress (for model [16]), or at vanishing logarithm of the mean stress normalised by the reference stress (for model [8]), denoted here as $e_{p 0}$. In this way, extended SOM-states which include void ratio can be defined (Fig. 2). Critical states can be considered as an example of extended SOM-states for strain paths with $\operatorname{tr} \mathbf{D}=0$, where $\mathrm{SOM}$ stress ratio follows from critical friction angle $\varphi_{c}$.
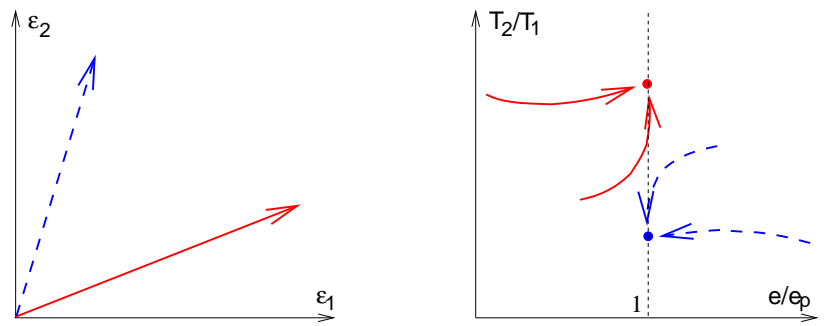

Fig. 2. Extended SOM-behaviour including void ratio

\section{Limit state (swept-out-memory) conditions}

As introduced in the previous section, at extended swept-out-memory states the stress rate tensor $\mathbf{T}$ has the same direction as the stress tensor $\mathbf{T}$ and the pyknotropy factor $f_{d}$ is constant $\left(\dot{f}_{d}=0\right)$ for one particular direction of 
stretching $\overrightarrow{\mathbf{D}}$. In the present developemnts, we will search for the value of the pyknotropy factor $f_{d}$ and direction of stretching $\overrightarrow{\mathbf{D}}$ which correspond to swept-out-memory states for the given stress state $\mathbf{T}$. The rate formulation of the considered hypoplastic equations reads

$$
\stackrel{\circ}{\mathbf{T}}=f_{s} \mathcal{L}: \mathbf{D}+f_{s} f_{d} \mathbf{N}\|\mathbf{D}\|
$$

which may be, without loss of generality, simplified by assuming $f_{s}$ being part of constitutive tensors $\mathcal{L}$ and $\mathbf{N}$ and taking $\|\mathbf{D}\|=1$ (considered models are rate independent - positively homogeneous of degree one in $\mathbf{D}$ ). Therefore

$$
\stackrel{\circ}{\mathbf{T}}=\mathcal{L}: \overrightarrow{\mathbf{D}}+f_{d} \mathbf{N}
$$

At swept-out-memory conditions $\stackrel{\mathbf{T}}{\|} \mathbf{T}$, so we may introduce a scalar multiplier $\gamma$ such that

$$
\stackrel{\circ}{\mathbf{T}}=\gamma \overrightarrow{\mathbf{T}}
$$

Therefore, swept-out-memory conditions are described by

$$
\gamma \overrightarrow{\mathbf{T}}=\mathcal{L}: \overrightarrow{\mathbf{D}}+f_{d} \mathbf{N}
$$

with

$$
\dot{f}_{d}=0
$$

Note that limit states calculated according to Eqs (8) and (9) include also proportional strain paths with $\operatorname{tr} \overrightarrow{\mathbf{D}}>0(\gamma<0)$.

\subsection{Hypoplastic model for clays}

Let us now solve the Eqn. 8 for the hypoplastic constitutive model for clays. The pyknotropy factor $f_{d}$ of this model is defined as

$$
f_{d}=\left(\frac{2 p}{p_{e}^{*}}\right)^{\alpha}
$$

where $p$ is the mean stress $p=-\operatorname{tr} \mathbf{T} / 3$ and $p_{e}^{*}$ is the equivalent pressure on the isotropic normal compression line, which is defined to be linear in the $\ln p: \ln (1+e)$ space and follows [1]

$$
\ln (1+e)=N-\lambda^{*} \ln \left(\frac{p_{e}^{*}}{p_{r}}\right)
$$

with $p_{r}$ being the reference stress of $1 \mathrm{kPa}$. Comparison of (10) and (11) reveals that $\dot{f}_{d}=0$ is satisfied for any line defined by

$$
\ln (1+e)=\text { const. }-\lambda^{*} \ln \left(\frac{p}{p_{r}}\right)
$$


Time-differentiation of (12) yields the rate formulation of normal compression lines, as defined in Sec. 3

$$
\frac{\dot{e}}{1+e}=-\frac{\lambda^{*}}{p} \dot{p}
$$

Because

$$
\dot{e}=(1+e) \operatorname{tr} \overrightarrow{\mathbf{D}}
$$

we have

$$
\operatorname{tr} \overrightarrow{\mathbf{D}}=-\frac{\lambda^{*}}{p} \dot{p}=-\lambda^{*} \frac{\operatorname{tr}(\gamma \overrightarrow{\mathbf{T}})}{\operatorname{tr} \mathbf{T}}=-\frac{\lambda^{*} \gamma}{\|\mathbf{T}\|}
$$

from which follows the expression for the scalar multiplier $\gamma$

$$
\gamma=-\|\mathbf{T}\| \frac{\operatorname{tr} \overrightarrow{\mathbf{D}}}{\lambda^{*}}
$$

We see that in the hypoplastic model for clays $\gamma$ is independent of void ratio (independent of the actual value of the pyknotropy factor $f_{d}$ ), which significantly simplifies derivation of the mathematical expression for swept-outmemory states.

Eqs. (16) and (8) may be combined

$$
-\frac{\mathbf{T}}{\lambda^{*}} \operatorname{tr} \overrightarrow{\mathbf{D}}=\mathcal{L}: \overrightarrow{\mathbf{D}}+f_{d} \mathbf{N}
$$

To solve this equation for $\overrightarrow{\mathbf{D}}$ and $f_{d}$, we introduce the fourth order tensor $\mathcal{A}$

$$
\mathcal{A}=\mathcal{L}+\frac{1}{\lambda^{*}} \mathbf{T} \otimes \mathbf{1}
$$

such that

$$
\mathcal{A}: \overrightarrow{\mathbf{D}}=\mathcal{L}: \overrightarrow{\mathbf{D}}+\frac{\mathbf{T}}{\lambda^{*}} \operatorname{tr} \overrightarrow{\mathbf{D}}
$$

holds. Eqn. (17) may be therefore written

$$
\mathcal{A}: \overrightarrow{\mathbf{D}}+f_{d} \mathbf{N}=0
$$

Since $\|\overrightarrow{\mathbf{D}}\|=1$, we get

$$
f_{d}=\left\|\mathcal{A}^{-1}: \mathbf{N}\right\|^{-1}
$$

and

$$
\overrightarrow{\mathbf{D}}=-\frac{\mathcal{A}^{-1}: \mathbf{N}}{\left\|\mathcal{A}^{-1}: \mathbf{N}\right\|}
$$

so we have a direction of stretching $\overrightarrow{\mathbf{D}}$ and the value of pyknotropy factor $f_{d}$ at swept-out-memory surface for any stress level T. Graphical representation of Eqs. (21) and (22) is demonstrated in Sec. 5. 


\subsection{Hypoplastic model for granular materials}

Solution of Eqns. (8) and (9) is less straightforward for the hypoplastic model for granular materials. In this case, the pyknotropy factor is defined as

$$
f_{d}=\left(\frac{e-e_{d}}{e_{c}-e_{d}}\right)^{\alpha}
$$

where $e_{c}$ and $e_{d}$ are characteristic void ratios which evolve with the mean stress according to

$$
\frac{e_{c}}{e_{c 0}}=\frac{e_{d}}{e_{d 0}}=\exp \left[-\left(\frac{3 p}{h_{s}}\right)^{n}\right]
$$

It follows from (23) and (24) that the pyknotropy factor of a hypoplastic model for granular materials is constant along any line defined by

$$
e=\text { (const.) } \exp \left[-\left(\frac{3 p}{h_{s}}\right)^{n}\right]
$$

which leads after time differentiation to

$$
\frac{\dot{e}}{e}=\frac{n}{h_{s}} \operatorname{tr} \stackrel{\circ}{\mathbf{T}}\left(\frac{3 p}{h_{s}}\right)^{(n-1)}
$$

By combining Eqs. (26) with (14) and (7) we have

$$
\operatorname{tr} \overrightarrow{\mathbf{D}}\left(\frac{1+e}{e}\right)=\gamma \frac{n}{h_{s}} \operatorname{tr} \overrightarrow{\mathbf{T}}\left(\frac{3 p}{h_{s}}\right)^{(n-1)}
$$

We see that the value of the scalar multiplier $\gamma$ of a hypoplastic model for granular materials depends on the void ratio, so also on the actual value of the pyknotropy factor $f_{d}$. To solve the set of Eqs. (27) and (8) for $\overrightarrow{\mathbf{D}}$ and $f_{d}$, we extract $\overrightarrow{\mathbf{D}}$ from Eq. (8) so we have

$$
\overrightarrow{\mathbf{D}}=\gamma\left(\mathcal{L}^{-1}: \overrightarrow{\mathbf{T}}\right)-f_{d}\left(\mathcal{L}^{-1}: \mathbf{N}\right)
$$

Combination of (28) and (27) yields the first equation relating $f_{d}$ and $\gamma$ which reads

$$
\gamma=-\frac{\left(\frac{1+e}{e}\right) \operatorname{tr} \mathbf{B}}{G-\left(\frac{1+e}{e}\right) \operatorname{tr} \mathbf{C}} f_{d}
$$

with

$$
\begin{aligned}
\mathbf{B} & =\mathcal{L}^{-1}: \mathbf{N} \\
\mathbf{C} & =\mathcal{L}^{-1}: \overrightarrow{\mathbf{T}} \\
G & =\frac{n}{h_{s}} \operatorname{tr} \overrightarrow{\mathbf{T}}\left(\frac{3 p}{h_{s}}\right)^{(n-1)}
\end{aligned}
$$


The second necessary equation is found by taking norm of (28). Because $\|\overrightarrow{\mathbf{D}}\|=1$ we have

$$
1=\|\mathbf{B}\|^{2} f_{d}^{2}+\|\mathbf{C}\|^{2} \gamma^{2}-2(\mathbf{B}: \mathbf{C}) f_{d} \gamma
$$

Combination of (29) and (33) yields an implicit equation for $f_{d}$

$$
f_{d}=\sqrt{\left[\|\mathbf{B}\|^{2}+\left(\frac{\|\mathbf{C}\|\left(\frac{1+e}{e}\right) \operatorname{tr} \mathbf{B}}{G-\left(\frac{1+e}{e}\right) \operatorname{tr} \mathbf{C}}\right)^{2}+\frac{2(\mathbf{B}: \mathbf{C}) \operatorname{tr} \mathbf{B}\left(\frac{1+e}{e}\right)}{G-\left(\frac{1+e}{e}\right) \operatorname{tr} \mathbf{C}}\right]^{-1}}
$$

where (from (23))

$$
e=f_{d}^{(1 / \alpha)}\left(e_{c}-e_{d}\right)+e_{d}
$$

Therefore, for hypoplastic model for granular materials explicit formulation for $\overrightarrow{\mathbf{D}}, f_{d}$ and $\gamma$ cannot be found. Eqn. (34) may be, however, solved numerically. We search for corresponding $e$ and $f_{d}$, while takning into account that also $f_{s}$ (and, therefore, also $\mathcal{L}$ and $\mathbf{N}$ which include $f_{s}$ in present developments) changes with $e$. Results are demonstrated graphically in Sec. 5 .

\section{Graphical representation}

Graphical representation of outlined equations for limit states is described in detail by Mašín and Gudehus [10]. State limits of both hypoplastic models for clays and for granular materials may be expressed by a graph relating $\overrightarrow{\mathbf{D}}$ and $\overrightarrow{\mathbf{T}}$. For axisymmetric states we define in Rendulic plane angles $\psi_{\sigma}$ and $\psi_{\dot{\epsilon}}$ according to Fig. 3. Special directions in the Rendulic plane of strain rate space

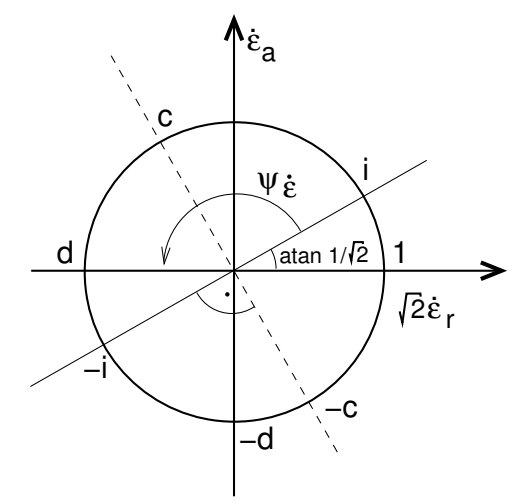

Fig. 3. Definition of angle $\psi_{\dot{\epsilon}}$ for axisymmetric states in Rendulic plane of $\boldsymbol{\epsilon}$ [10]. Angle $\psi_{\sigma}$ is defined accordingly in the Rendulic plane of $\boldsymbol{\sigma}$.

are denoted by "i" and "-i" (for isotropic compression and extension), "c" and 
"-c" (for isochoric compression and extension) and "d" and "-d" (for extension with $\epsilon_{a}=0$ and $\epsilon_{r}=0$ respectively). $\psi_{\sigma}$ versus $\psi_{\dot{\epsilon}}$ plots for both models are shown in Fig. 4. Parameters used for simulations with a hypoplastic model for granular materials follow from [5] (Tab. 1), parameters of a hypoplastic model for clays have been compiled from different publications (see Tab. 2 for references).

Table 1. Parameters of a hypoplastic model for granular materials [5]

\begin{tabular}{c|ccccccccc}
\hline soil & $\varphi_{c}\left[^{\circ}\right]$ & $h_{s}[\mathrm{MPa}]$ & $n$ & $e_{d 0}$ & $e_{c 0}$ & $e_{i 0}$ & $\alpha$ & $\beta$ \\
\hline Zbraslav sand & 31 & 5700 & 0.25 & 0.52 & 0.82 & 0.95 & 0.13 & 1.00 \\
Toyoura sand & 30 & 2600 & 0.27 & 0.61 & 0.98 & 1.10 & 0.18 & 1.00 \\
Hochstetten sand & 33 & 1500 & 0.28 & 0.55 & 0.95 & 1.05 & 0.25 & 1.50 \\
Schlabendorf sand & 33 & 1600 & 0.19 & 0.44 & 0.85 & 1.00 & 0.25 & 1.00 \\
Hostun sand & 31 & 1000 & 0.29 & 0.61 & 0.91 & 1.09 & 0.13 & 2.00 \\
Karlsruhe sand & 30 & 5800 & 0.28 & 0.53 & 0.84 & 1.00 & 0.13 & 1.05 \\
Ottawa sand & 30 & 4900 & 0.29 & 0.49 & 0.76 & 0.88 & 0.10 & 1.00 \\
Ticino sand & 31 & 5800 & 0.31 & 0.60 & 0.93 & 1.05 & 0.20 & 1.00 \\
SLB sand & 30 & 8900 & 0.33 & 0.49 & 0.79 & 0.90 & 0.14 & 1.00 \\
\hline
\end{tabular}

Table 2. Parameters of a hypoplastic model for clays

\begin{tabular}{cc|ccccc}
\hline soil & reference $\mid \varphi_{c}\left[^{\circ}\right]$ & $\lambda^{*}$ & $\kappa^{*}$ & $N$ & $r$ \\
\hline London clay & {$[8]$} & 22.6 & 0.11 & 0.014 & 1.375 & 0.4 \\
Beaucaire marl & {$[12]$} & 33 & 0.057 & 0.007 & 0.85 & 0.4 \\
Pisa clay & {$[9]$} & 21.9 & 0.14 & 0.005 & 1.56 & 0.2 \\
Bothkennar clay & {$[9]$} & 35 & 0.119 & 0.002 & 1.344 & 0.05 \\
\hline
\end{tabular}

Second plot used to characterise limit state conditions relates $\psi_{\sigma}$ with a function of the pyknotropy factor $f_{d}$ at limit states. A suitable quantities follow from the formulation of pyknotropy factors of the two models considered (Eq. (10) and (23)). The influence of pyknotropy for the model for granular materials is characterised by a relative void ratio $r_{e}$ defined as

$$
r_{e}=\frac{e-e_{d}}{e_{c}-e_{d}}
$$

whereas the degree of overconsolidation for the model for clays may be charactersised by overconsolidation ratio $(O C R)$, defined as

$$
O C R=\frac{p_{e}^{*}}{p}
$$




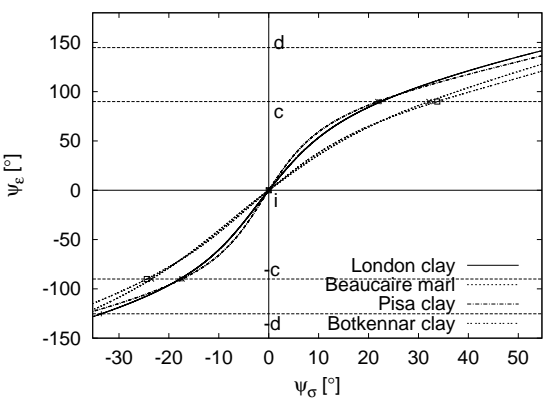

(a)

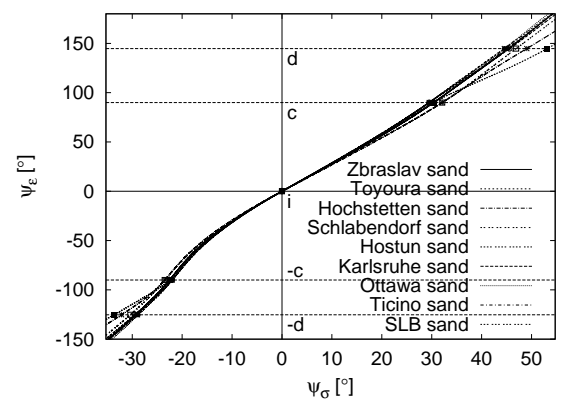

(b)

Fig. 4. $\psi_{\sigma}: \psi_{\dot{\epsilon}}$ plots for the hypoplastic model for clays (a) and hypoplastic model for granular materials (b).

Graphs relating $\psi_{\sigma}$ with $r_{e}$ for the model for granular materials and $\psi_{\sigma}$ with $O C R$ for the model for clays are in Fig. 5.

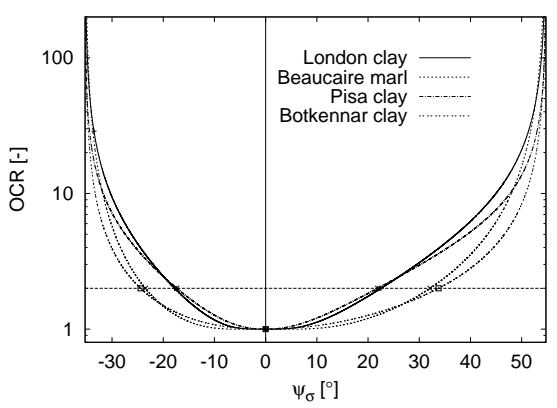

(a)

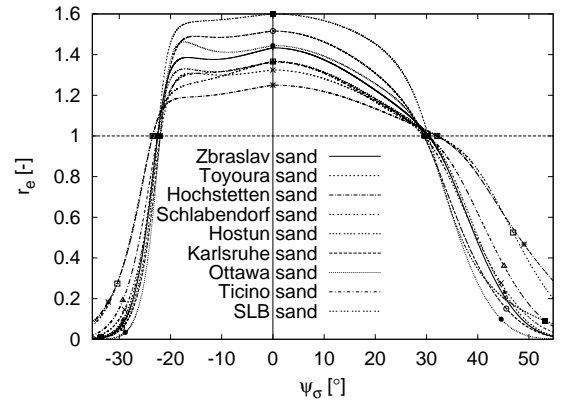

(b)

Fig. 5. $\psi_{\sigma}: O C R$ and $\psi_{\sigma}: r_{e}$ plots for the hypoplastic model for clays (a) and hypoplastic model for granular materials (b)

\section{Concluding remarks}

As pointed out in the introduction, limit state conditions are important aspects of soil behaviour which must be predicted by advanced constitutive models. The paper presented mathematical derivation for swept-out-memory (limit) states of the two hypoplastic constitutive models. It has been shown that the hypoplastic model for clays allows for an explicit formulation of limit state conditions. The expression for limit state conditions may be evaluated also for a hypoplastic model for granular materials. In this case, however, the 
analytical solution of the problem is not available and governing equations must be solved numerically. An experimental check of the limit state loci predicted by the models is a matter of further research. Presented derivations are important for incorporating structural effects into hypoplastic models, as demonstrated in [9] by formulating a hypoplastic model for structured clays.

\section{Acknowledgement}

The first author is grateful for the financial support by the research grants SSPI-CT-2003-501837-NOAH'S ARK under the EC 6th FP and GACR 205/03/1467.

\section{References}

1. R. Butterfield. A natural compression law for soils. Géotechnique, 29(4):469480, 1979.

2. G. Gudehus. Attractors for granular sorage and flow. In $3^{\text {rd }}$ European Symposium - Storage and Flow of Particulate Solids, Paper for the conf. 'Partec 95', pages 333-345, 1995.

3. G. Gudehus. A comprehensive constitutive equation for granular materials. Soils and Foundations, 36(1):1-12, 1996.

4. G. Gudehus. A visco-hypoplastic constitutive relation for soft soils. Soils and Foundations, 44(4):11-25, 2004.

5. I. Herle. Hypoplastizität und granulometrie of einfacher korngerüste. $\mathrm{PhD}$ thesis, Universität Fridericiana in Karlsruhe, 1997.

6. I. Herle and D. Kolymbas. Hypoplasticity for soils with low friction angles. Computers and Geotechnics, 31(5):365-373, 2004.

7. D. Kolymbas. An outline of hypoplasticity. Archive of Applied Mechanics, 61:143-151, 1991.

8. D. Mašín. A hypoplastic constitutive model for clays. International Journal for Numerical and Analytical Methods in Geomechanics, 29(4):311-336, 2005.

9. D. Mašín. A hypoplastic constitutive model for clays with meta-stable structure. (in preparation), 2005.

10. D. Mašín and G. Gudehus. Graphical representation of rate independent constitutive relations for saturated clays. Soils and Foundations (in preparation), 2006.

11. D. Mašín and I. Herle. State boundary surface of a hypoplastic model for clays. Computers and Geotechnics (submitted for publication), 2005.

12. D. Mašín, C. Tamagnini, G. Viggiani, and D. Costanzo. Directional response of a reconstituted fine granied soil. part II: Performance of different constitutive models. International Journal for Numerical and Analytical Methods in Geomechanics (submitted for publication), 2005.

13. A. Niemunis. A visco-hypoplastic model for clay and its FE implementation. In Resultats recents en mechanique des sols et des roches XI Colloque FrancoPolonais. Gdańsk, 1996. 
14. A. Niemunis. Extended hypoplastic models for soils. Habilitation thesis, RuhrUniversity, Bochum, 2002.

15. C. Tamagnini, G. Viggiani, and R. Chambon. A review of two different approaches to hypoplasticity. In D. Kolymbas, editor, Constitutive modelling of granular materials, pages 107-144. Springer, 1999, 1999.

16. P. A. von Wolffersdorff. A hypoplastic relation for granular materials with a predefined limit state surface. Mechanics of Cohesive-Frictional Materials, 1:251-271, 1996.

\section{Appendix A}

The mathematical structure of the hypoplastic model for clays is discussed in detail in [8]. The constitutive equation in rate form reads:

$$
\stackrel{\circ}{\mathbf{T}}=f_{s} \mathcal{L}: \mathbf{D}+f_{s} f_{d} \mathbf{N}\|\mathbf{D}\|
$$

where:

$$
\mathcal{L}=3\left(c_{1} \mathcal{I}+c_{2} a^{2} \hat{\mathbf{T}} \otimes \hat{\mathbf{T}}\right) \quad \mathbf{N}=\mathcal{L}:\left(-Y \frac{\mathbf{m}}{\|\mathbf{m}\|}\right) \quad \hat{\mathbf{T}}:=\frac{\mathbf{T}}{\operatorname{tr} \mathbf{T}}
$$

$\mathbf{1}$ is the second-order identity tensor and $\mathcal{I}$ is the fourth-order identity tensor, with components:

$$
(\mathcal{I})_{i j k l}:=\frac{1}{2}\left(1_{i k} 1_{j l}+1_{i l} 1_{j k}\right)
$$

In eq. (38), the functions $f_{s}(\operatorname{tr} \mathbf{T})$ (barotropy factor) and $f_{d}(\operatorname{tr} \mathbf{T}$, e) (pyknotropy factor) are given by:

$$
f_{s}=-\frac{\operatorname{tr} \mathbf{T}}{\lambda^{*}}\left(3+a^{2}-2^{\alpha} a \sqrt{3}\right)^{-1} f_{d}=\left[-\frac{2 \operatorname{tr} \mathbf{T}}{3 p_{r}} \exp \left(\frac{\ln (1+e)-N}{\lambda^{*}}\right)\right]^{\alpha}
$$

where $p_{r}$ is the reference stress $1 \mathrm{kPa}$. The scalar function $Y$ and second-order tensor $\mathrm{m}$ appearing in Eq. (39) are given, respectively, by:

$$
Y=\left(\frac{\sqrt{3} a}{3+a^{2}}-1\right) \frac{\left(I_{1} I_{2}+9 I_{3}\right)\left(1-\sin ^{2} \varphi_{c}\right)}{8 I_{3} \sin ^{2} \varphi_{c}}+\frac{\sqrt{3} a}{3+a^{2}}
$$

in which:

$$
I_{1}:=\operatorname{tr} \mathbf{T} \quad I_{2}:=\frac{1}{2}\left[\mathbf{T}: \mathbf{T}-\left(I_{1}\right)^{2}\right] \quad I_{3}:=\operatorname{det} \mathbf{T}
$$

and:

in which:

$$
\mathbf{m}=-\frac{a}{F}\left[\hat{\mathbf{T}}+\hat{\mathbf{T}}^{*}-\frac{\hat{\mathbf{T}}}{3}\left(\frac{6 \hat{\mathbf{T}}: \hat{\mathbf{T}}-1}{(F / a)^{2}+\hat{\mathbf{T}}: \hat{\mathbf{T}}}\right)\right]
$$

$$
\begin{array}{rlrl}
\hat{\mathbf{T}}^{*} & =\hat{\mathbf{T}}-\frac{\mathbf{1}}{3} \quad F & =\sqrt{\frac{1}{8} \tan ^{2} \psi+\frac{2-\tan ^{2} \psi}{2+\sqrt{2} \tan \psi \cos 3 \theta}}-\frac{1}{2 \sqrt{2}} \tan \psi \\
\tan \psi & =\sqrt{3}\left\|\hat{\mathbf{T}}^{*}\right\| & \cos 3 \theta & =-\sqrt{6} \frac{\operatorname{tr}\left(\hat{\mathbf{T}}^{*} \cdot \hat{\mathbf{T}}^{*} \cdot \hat{\mathbf{T}}^{*}\right)}{\left(\hat{\mathbf{T}}^{*}: \hat{\mathbf{T}}^{*}\right)^{3 / 2}}
\end{array}
$$


Finally, the scalars $a, \alpha, c_{1}$ and $c_{2}$ appearing in eqs. (39)-(43), are given as functions of the material parameters $\varphi_{c}, \lambda^{*}, \kappa^{*}$ and $r$ by the following relations:

$$
\begin{aligned}
a & =\frac{\sqrt{3}\left(3-\sin \varphi_{c}\right)}{2 \sqrt{2} \sin \varphi_{c}} & \alpha & =\frac{1}{\ln 2} \ln \left[\frac{\lambda^{*}-\kappa^{*}}{\lambda^{*}+\kappa^{*}}\left(\frac{3+a^{2}}{a \sqrt{3}}\right)\right] \\
c_{1} & =\frac{2\left(3+a^{2}-2^{\alpha} a \sqrt{3}\right)}{9 r} & c_{2} & =1+\left(1-c_{1}\right) \frac{3}{a^{2}}
\end{aligned}
$$

The model requires five constitutive parameters, namely $\varphi_{c}, \lambda^{*}, \kappa^{*}, N$ and $r$.

\section{Appendix B}

This appendix summarizes mathematical formulation of a hypoplastic model for granular materials [16].

The model assumes the following stress-strain relation:

$$
\stackrel{\circ}{\mathbf{T}}=f_{s} \mathcal{L}: \mathbf{D}+f_{s} f_{d} \mathbf{N}\|\mathbf{D}\|
$$

with

$$
\begin{gathered}
\mathcal{L}=\frac{1}{\hat{\mathbf{T}}: \hat{\mathbf{T}}}\left(F^{2} \mathcal{I}+a^{2} \hat{\mathbf{T}} \otimes \hat{\mathbf{T}}\right) \\
\mathbf{N}=\frac{F a}{\hat{\mathbf{T}}: \hat{\mathbf{T}}}\left(\hat{\mathbf{T}}+\hat{\mathbf{T}}^{*}\right)
\end{gathered}
$$

where 1 is a second-order unity tensor, $\mathcal{I}_{i j k l}=\frac{1}{2}\left(1_{i k} 1_{j l}+1_{i l} 1_{j k}\right)$ is a fourth-order unity tensor and

$$
\begin{gathered}
\operatorname{tr} \mathbf{T}=\mathbf{T}: \mathbf{1}, \quad \hat{\mathbf{T}}=\mathbf{T} / \operatorname{tr} \mathbf{T}, \quad \hat{\mathbf{T}}^{*}=\hat{\mathbf{T}}-\mathbf{1} / 3 \\
a=\frac{\sqrt{3}\left(3-\sin \varphi_{c}\right)}{2 \sqrt{2} \sin \varphi_{c}}, \quad F=\sqrt{\frac{1}{8} \tan ^{2} \psi+\frac{2-\tan ^{2} \psi}{2+\sqrt{2} \tan \psi \cos 3 \theta}}-\frac{1}{2 \sqrt{2}} \tan \psi
\end{gathered}
$$

with

$$
\tan \psi=\sqrt{3}\left\|\hat{\mathbf{T}}^{*}\right\|, \quad \cos 3 \theta=-\sqrt{6}, \frac{\operatorname{tr}\left(\hat{\mathbf{T}}^{*} \cdot \hat{\mathbf{T}}^{*} \cdot \hat{\mathbf{T}}^{*}\right)}{\left[\hat{\mathbf{T}}^{*}: \hat{\mathbf{T}}^{*}\right]^{3 / 2}}
$$

The scalar factors $f_{s}$ and $f_{d}$ take into account the influence of mean pressure and density,

$$
\begin{gathered}
f_{s}=\frac{h_{s}}{n}\left(\frac{e_{i}}{e}\right)^{\beta} \frac{1+e_{i}}{e_{i}}\left(\frac{-\operatorname{tr} \mathbf{T}}{h_{s}}\right)^{1-n}\left[3+a^{2}-a \sqrt{3}\left(\frac{e_{i 0}-e_{d 0}}{e_{c 0}-e_{d 0}}\right)^{\alpha}\right]^{-1} \\
f_{d}=\left(\frac{e-e_{d}}{e_{c}-e_{d}}\right)^{\alpha}
\end{gathered}
$$

The characteristic void ratios $-e_{i}, e_{c}$ and $e_{d}$ decrease with the mean pressure according to the relation

$$
\frac{e_{i}}{e_{i 0}}=\frac{e_{c}}{e_{c 0}}=\frac{e_{d}}{e_{d 0}}=\exp \left[-\left(\frac{-\operatorname{tr} \mathbf{T}}{h_{s}}\right)^{n}\right]
$$

The model requires 8 parameters: $\phi_{c}, h_{s}, n, e_{d 0}, e_{c 0}, e_{i 0}, \alpha$ and $\beta$. 